Claremont Colleges

Scholarship@ Claremont

All HMC Faculty Publications and Research

HMC Faculty Scholarship

$10-1-2001$

\title{
Distinguishing Surface and Bulk Contributions to Third-Harmonic Generation in Silicon
}

Peter N. Saeta

Harvey Mudd College

Nathan A. Miller'01

Harvey Mudd College

\section{Recommended Citation}

"Distinguishing surface and bulk contributions to third-harmonic generation in silicon," P. N. Saeta and N. A. Miller*, Appl. Phys. Lett. 79, 2704 (2001). doi: 10.1063/1.1412434

This Article is brought to you for free and open access by the HMC Faculty Scholarship at Scholarship @ Claremont. It has been accepted for inclusion in All HMC Faculty Publications and Research by an authorized administrator of Scholarship @ Claremont. For more information, please contact scholarship@cuc.claremont.edu. 


\section{AIP Applied Physics \\ Letters}

\section{Distinguishing surface and bulk contributions to third-harmonic generation in silicon}

P. N. Saeta and N. A. Miller

Citation: Appl. Phys. Lett. 79, 2704 (2001); doi: 10.1063/1.1412434

View online: http://dx.doi.org/10.1063/1.1412434

View Table of Contents: http://apl.aip.org/resource/1/APPLAB/v79/i17

Published by the AIP Publishing LLC.

Additional information on Appl. Phys. Lett.

Journal Homepage: http://apl.aip.org/

Journal Information: http://apl.aip.org/about/about_the_journal

Top downloads: http://apl.aip.org/features/most_downloaded

Information for Authors: http://apl.aip.org/authors

\section{ADVERTISEMENT}
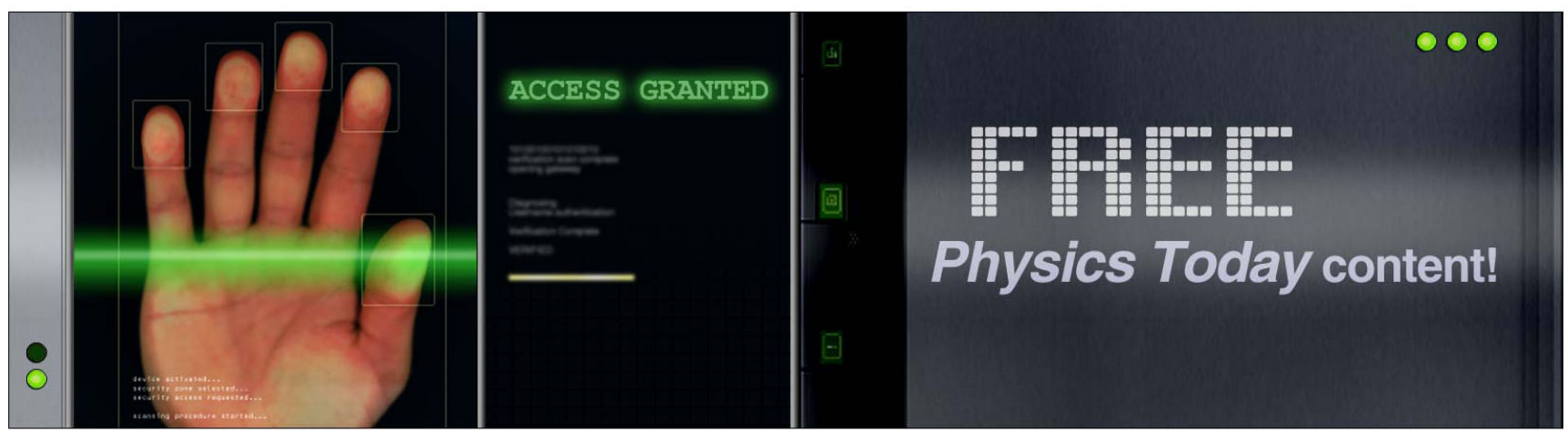


\title{
Distinguishing surface and bulk contributions to third-harmonic generation in silicon
}

\author{
P. N. Saeta ${ }^{a)}$ and N. A. Miller \\ Physics Department, Harvey Mudd College, Claremont, California 91711
}

(Received 25 June 2001; accepted for publication 13 August 2001)

\begin{abstract}
We report measurements of third-harmonic generation from ultrathin crystalline silicon layers of gradually varying thickness. Both the angular and thickness dependence of the third-harmonic light generated in transmission at normal incidence are consistent with negligible surface contribution to third-harmonic generation in silicon, even under tight focusing. This work illustrates a method for distinguishing surface and bulk contributions to harmonic generation. (c) 2001 American Institute of Physics. [DOI: 10.1063/1.1412434]
\end{abstract}

Nonlinear optical methods of studying surfaces and interfaces are attractive because they are noninvasive and nondestructive, can be used in a vacuum and in air, have enhanced sensitivity to atomic structure and bonding at interfaces, and can be used in real time. ${ }^{1-6}$ In centrosymmetric materials, such as silicon, even-order optical processes are forbidden to lowest order in perturbation theory, and this symmetry gives rise to the relative enhancement of secondharmonic generation at surfaces and interfaces, where the bulk symmetry is broken. ${ }^{7}$ Inversion symmetry does not suppress third-harmonic generation (THG), however. Thus, one expects that, barring strong surface enhancement, the bulk contribution should exceed the surface contribution by roughly $\ell / a$, where $\ell$ is the shorter of the coherence length and the escape depth of the third harmonic, and $a$ is the lattice constant. ${ }^{8}$ Even for strongly absorbing materials such as silicon, the number of bulk atoms participating in THG should greatly exceed the number of surface atoms, making THG an essentially bulk phenomenon.

Recent work by Tsang, ${ }^{9,10}$ however, appears at odds with this interpretation. Using tightly focused femtosecond pulses from an unamplified Ti:sapphire oscillator, Refs. 9 and 10 investigated the dependence of THG on focusing conditions in both transparent and opaque materials. Transparent materials showed a tremendous enhancement in transmitted THG on focusing at surfaces and interfaces. With tightly focused beams, one expects a strong suppression of THG caused by the Gouy phase anomaly: ${ }^{11}$ as the beam passes through focus, the phase of the third-harmonic (TH) source polarization varies by $3 \pi$, thus spoiling phase matching. The significance of this effect can be diminished by breaking the symmetry of the focal region with an interface, ${ }^{12}$ although Tsang reported that the phase shift effect was insufficient to account for observations. ${ }^{9}$ Rather, Tsang attributed the enhancement to a significant surface contribution to THG. Such a surface polarization could arise from the strong electric field gradient near the interface between two dissimilar dielectric media.

To investigate the possible surface enhancement to THG at $\mathrm{Si}-\mathrm{SiO}_{2}$ interfaces, we have measured THG from ultrathin silicon layers as a function of $\mathrm{Si}$ layer thickness for thick-

${ }^{\text {a)} E l e c t r o n i c ~ m a i l: ~ s a e t a @ h m c . e d u ~}$ nesses small and large compared to the absorption depth of the third harmonic. These measurements constitute a means to distinguish surface and bulk contributions to harmonic generation and show unambiguously that THG is bulk dominant in silicon.

Samples were prepared from UNIBOND silicon-oninsulator substrates made by Soitec, which had a 200-nm $c$ - $\mathrm{Si}(001)$ layer press bonded to a fused silica substrate. ${ }^{13,14}$ Pieces were oxidized in dry $\mathrm{O}_{2}$ in a quartz tube furnace at temperatures between $950^{\circ} \mathrm{C}$ and $1050{ }^{\circ} \mathrm{C}$. A spatial temperature gradient was used to produce a silicon layer of gently tapered thickness ${ }^{15,16}$ varying by approximately $30 \mathrm{~nm}$ over a lateral distance of $\sim 30 \mathrm{~mm}$. The structure is illustrated in Fig. 1(a). To explore layer thicknesses $d$ from 0 to $120 \mathrm{~nm}$, several different samples were prepared, with overlapping thickness ranges. The gradual taper of the layer permits the thickness dependence of the THG to be investigated on a single sample with consistent orientation and oxide conditions.

(a)
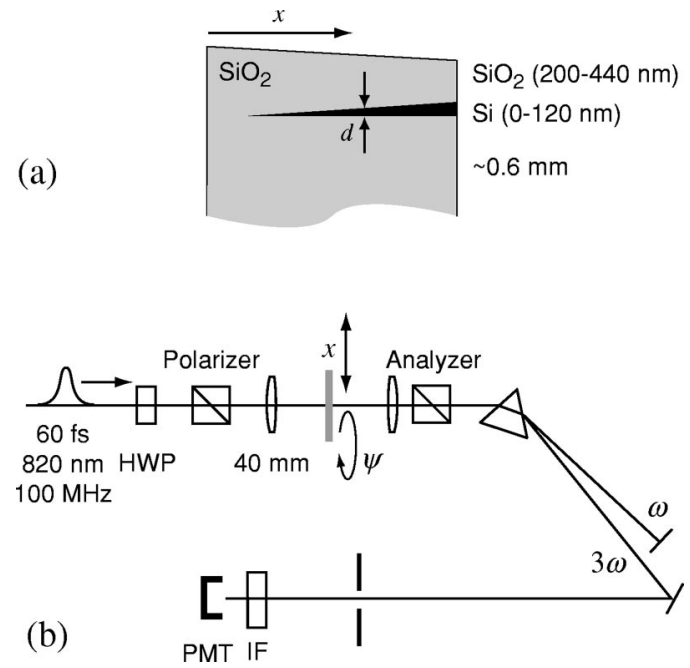

FIG. 1. (a) A representation of the sample structure. The silicon layer thickness $d$ (and upper oxide thickness) varied smoothly with position $x$ along the sample in the direction of translation. The wedge angle of the silicon layer was typically of order $1 \mu \mathrm{rad}$. (b) The experimental setup used to measure THG in transmission through transparent layers. The sample was mounted either to a rotation stage, allowing us to measure the azimuthal dependence of the THG at a particular spot on the sample, or to a translation stage, allowing us to measure the dependence of the THG on silicon layer thickness. 


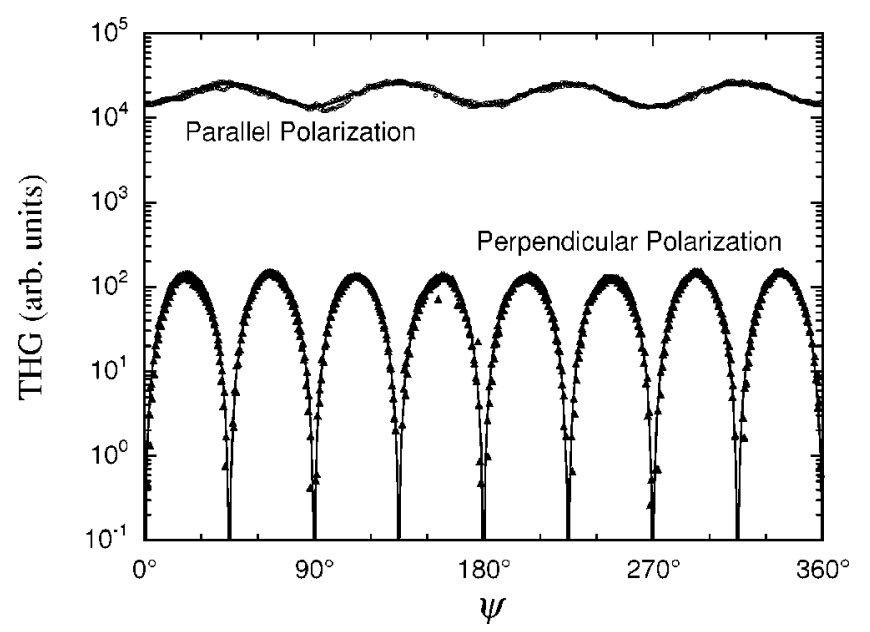

FIG. 2. Normal-incidence transmitted THG as a function of sample rotation $\psi$ with respect to a (100) direction. The data are consistent with the expected symmetry of the bulk silicon crystal, as shown in the fitted curves.

The thickness profile of the silicon layer and oxide overlayer were determined by measuring transmission spectra with a spectrophotometer in the range $200-800 \mathrm{~nm}$ and fitting to the thin-film equations. ${ }^{17}$ With decreasing $d$ below 6 $\mathrm{nm}$ departures from the dielectric function of bulk $c$-Si (Ref. 17) were observed, particularly in the neighborhood of the $E_{1}$ point. In this thickness range, quantum confinement effects significantly modify the dielectric function. However, good fits were obtained using a thickness-dependent semiempirical model of the dielectric function of $c$-Si layers. ${ }^{18}$

The THG properties of the silicon layers were studied using a 100-MHz mode-locked Ti:sapphire laser (ClarkMXR NJA-5) producing 60-fs pulses centered at $820 \mathrm{~nm}$, and the setup illustrated in Fig. 1(b). The beam was focused with a $40 \mathrm{~mm}$ focal length lens to a spot radius of $7 \mu \mathrm{m}$; the peak intensity was below $20 \mathrm{GW} / \mathrm{cm}^{2}$. The transmitted THG was measured both as a function of sample rotation at fixed sample position, using a rotation stage, and as a function of position for fixed sample rotation, using a translation stage. A thermoelectrically cooled photomultiplier detected the $\mathrm{TH}$ photons. For the measurements reported here, the fundamental beam was incident along the normal and the transmitted THG was collimated, analyzed with a second polarizer, and separated from the fundamental with an equilateral fused silica prism. Stray fundamental light was rejected by a 20-nm bandpass interference filter centered at $266 \mathrm{~nm}$. Care was taken to align the sample plane with the translation axis to within $2 \mathrm{mrad}$ to avoid changes in focusing at the surface when measuring the thickness dependence of the THG.

Rotational scans of the TH intensity polarized parallel and perpendicular to the incident field are shown in Fig. 2. The azimuthal angle $\psi$ is measured with respect to the (100) direction. The parallel-polarization data were obtained at a silicon thickness of $34 \mathrm{~nm}$; they were fitted to the expression

$$
\begin{aligned}
I_{\|}(3 \omega) & \propto\left[3 \chi_{1111}+3 \chi_{1212}-\left(3 \chi_{1212}-\chi_{1111}\right) \cos 4 \psi\right]^{2} \\
& \propto[(4+\sigma)-\sigma \cos 4 \psi]^{2},
\end{aligned}
$$

where $\sigma \equiv 3 \chi_{1212} / \chi_{1111}-1$ is the anisotropy parameter. ${ }^{19}$ The resulting value of $\sigma=0.71 \pm 0.01$ is consistent with previous work on bulk samples using 770-nm femtosecond pulses, ${ }^{9,20}$ and differs slightly from a value at $819 \mathrm{~nm}$ using

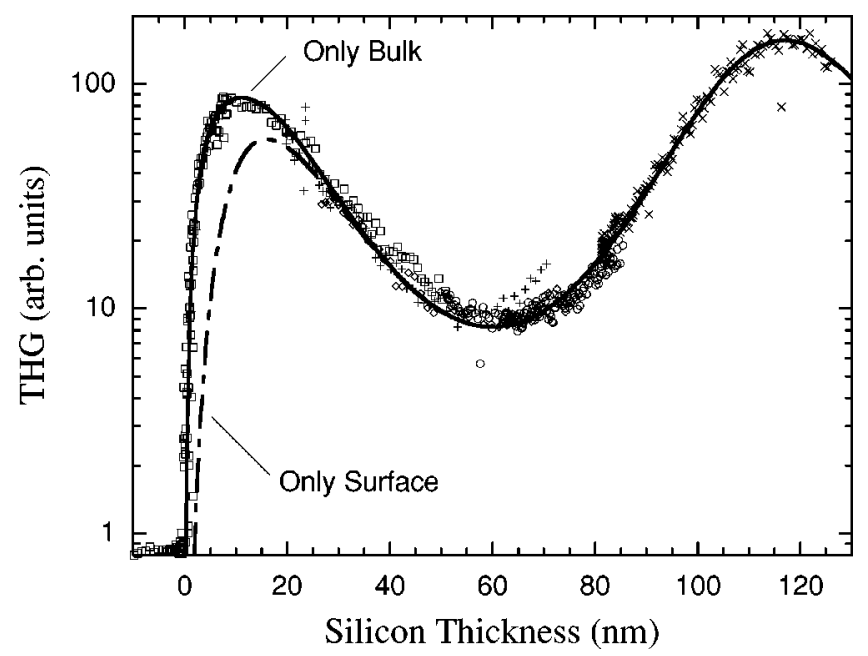

FIG. 3. Normal-incidence parallel-polarized THG as a function of silicon layer thickness. The data from five different samples are combined to span the range from 0 to $120 \mathrm{~nm}$. Each data set, indicated by a different symbol, has been scaled to match in regions of overlap, to account for variations in laser intensity. The solid curve is the calculated thickness dependence for pure bulk THG; the broken curve shows the thickness dependence of surface-dominant THG, scaled to match the bulk curve at large thickness.

nanosecond pulses. ${ }^{19}$ The perpendicular THG has the expected eight-fold dependence given by $I_{\perp}(3 \omega)$ $\propto \chi_{1111}^{2} \sin ^{2} 4 \psi$. The scan shown in Fig. 2 was taken at a layer thickness of $22 \mathrm{~nm}$; similar traces were obtained for both parallel and perpendicular polarization throughout the range of this sample, which was $0-35 \mathrm{~nm}$. The rotational THG scans thus confirm the bulk crystal symmetry and are consistent with, though not proof of, a bulk-dominant THG process in silicon, since the same azimuthal dependence would arise from surface-dominant THG.

We now show that the dependence of the TH signal on silicon layer thickness $d$ provides such proof. Figure 3 shows the parallel THG as a function of $d$ over the range $0 \leqslant d$ $\leqslant 120 \mathrm{~nm}$. Data from five different samples are combined in Fig. 3 by scaling to match in the regions of overlap, and the combined data set is compared to calculations of the $d$ dependence for bulk-dominant and surface-dominant THG. The data plotted at negative $d$ correspond to points on the thinnest sample past the end of the silicon film, and represent the background signal level. This signal is predominantly stray fundamental light leaking through the interference filter, as was confirmed by its linear dependence on pump intensity. It is more strongly suppressed in the rotational scans shown in Fig. 2 because of the greater path length between the dispersing prism and the detector in that setup.

The curves shown in Fig. 3 were calculated using a matrix formalism we have developed to account for the significant variation in the strength of the fundamental field in the silicon layer as a function of the thickness of both the upper oxide layer and the silicon layer itself. ${ }^{21}$ In brief, the linear problem is solved first for the incident conditions of interest, yielding the amplitude and phase of the forward- and backward-going fundamental waves in the silicon layer. From these, the polarization at $3 \omega$ is computed and used to solve the inhomogeneous wave equation,

$$
\nabla(\nabla \times \mathbf{E})-\nabla^{2} \mathbf{E}-N^{2} \widetilde{\Omega}^{2} \mathbf{E}=4 \pi \widetilde{\Omega}^{2} \mathbf{P}_{q} e^{i q z},
$$


where $N$ is the index of refraction at $3 \omega ; \mathbf{P}_{q}$ is the nonlinear polarization at $3 \omega$ having wave vector $q$ along the sample normal, which is along the $z$ axis; and $\widetilde{\Omega}=3 \omega / c$ is the vacuum wave vector at $3 \omega^{8,22}$

In the absence of reflections at the $\mathrm{Si}-\mathrm{SiO}_{2}$ interfaces, there is a single polarization term with $q=3 w=3 n k$, where $n$ is the refractive index at $\omega, k=2 \pi / \lambda$ is the magnitude of the vacuum wave vector of the fundamental beam, and we assume normal incidence. However, the high dielectric contrast between $\mathrm{Si}$ and $\mathrm{SiO}_{2}$ leads to significant reflections and strong modulation of the fundamental intensity in the silicon layer. At $d=60 \mathrm{~nm}$, for example, the fundamental field is only $61 \%$ of its peak value, which produces the pronounced dip in the detected THG shown in Fig. 3. Furthermore, the presence of significant backward-propagating waves gives rise to three additional bulk source polarizations, with $q$ $=w,-w$, and $-3 w$. These correspond to taking 2, 1 , and 0 photons from the incident wave (and 1, 2, and 3 photons from the reflected wave, respectively). Curiously, because of the great difference between $n(\omega)$ and $n(3 \omega)$ (3.68 vs $2.25+4.95 i)$, the nonlinear sources that combine one photon from one beam with two from the other are better phase matched than the "usual" source that takes all three from the incident beam. It is, therefore, essential to include these "mixed" sources in the calculation. Note, however, that because the silicon thickness is 1000 times smaller than the confocal parameter, the Gouy phase anomaly is negligible here.

The inhomogeneous waves from the four source polarizations combine with forward and backward freely propagating waves at $3 \omega$ in the layer, and with outgoing TH waves in the surrounding oxide layers. In the absence of surface source terms, the parallel components of electric and magnetic fields are continuous. If significant surface sources are present, these produce discontinuities in the parallel components of the harmonic fields at the interfaces. ${ }^{2,3}$ The boundary conditions permit the transmitted and reflected THG to be determined. The solid curve in Fig. 3 was computed assuming negligible surface sources. The agreement with the data throughout the 120-nm range of silicon layer thicknesses is excellent, including the range of thicknesses below $20 \mathrm{~nm}$. Essentially, the same behavior was observed with a tighter focusing $15-\mathrm{mm}$ focal-length objective.

The broken curve is calculated using no bulk sources, only surface sources. ${ }^{23}$ It has been scaled to agree with the bulk-only calculation for $d$ much greater than the attenuation length of the TH field, which is $\alpha^{-1}=8.8 \mathrm{~nm}$. For $d$ $\gg \alpha^{-1}$, any TH wave generated at the opposite interface is strongly attenuated in traversing the silicon layer. The dependence of the THG on $d$ then arises solely from the variations in the fundamental field strength inside the layer, caused by the multiple reflections of the virtually unattenuated fundamental wave. Hence, for $d \gg \alpha^{-1}$, surface and bulk sources are indistinguishable based on thickness. However, for small $d$, the difference between the surface and bulk sources is significant, with the surface-dominant THG rising considerably more slowly with silicon layer thickness. These data argue persuasively against significant surface enhancement to THG in silicon.

In summary, we have studied how transmitted THG depends on the thickness $d$ of a $\mathrm{Si}(001)$ layer surrounded by oxide. For $d$ large compared to the absorption depth of the $\mathrm{TH}$, the transmitted THG tracks the modulations in the intensity of the fundamental in the silicon layer, independent of the relative strength of surface to bulk sources. When $d$ is of the order of the absorption depth, however, the predictions of surface-dominant and bulk-dominant THG differ significantly, and the data demonstrate a negligible contribution from surface effects. These measurements illustrate an approach to distinguish surface and bulk contributions to harmonic generation with broad applicability. Experiments on the thickness dependence of second-harmonic generation in silicon are under way and open up a means to study a nonlocal nonlinear source that is inaccessible to single-beam experiments.

This work was supported by Harvey Mudd College and the Research Corporation (Cottrell College Science Award CC4262). The authors gratefully acknowledge assistance from P. D. Sparks, J. C. Eckert, T. D. Donnelly, A. W. Rollins, and M. J. Smith-Martinez.

${ }^{1}$ H. W. K. Tom, T. F. Heinz, and Y. R. Shen, Phys. Rev. Lett. 51, 1983 (1983).

${ }^{2}$ Y. R. Shen, The Principles of Nonlinear Optics (Wiley, New York, 1984).

${ }^{3}$ P. Guyot-Sionnest, W. Chen, and Y. R. Shen, Phys. Rev. B 33, 8254 (1986).

${ }^{4}$ H. Tom, G. Aumiller, and C. Brito-Cruz, Phys. Rev. Lett. 60, 1438 (1988).

${ }^{5}$ Y. R. Shen, Nature (London) 337, 519 (1989).

${ }^{6}$ J. I. Dadap, Z. Xu, X. F. Hu, and M. C. Downer, Phys. Rev. B 56, 13367 (1997).

${ }^{7}$ N. Bloembergen, R. Chang, S. Jha, and C. Lee, Phys. Rev. 174, 813 (1968).

${ }^{8}$ J. E. Sipe, D. J. Moss, and H. M. van Driel, Phys. Rev. B 35, 1129 (1987).

${ }^{9}$ T. Y. F. Tsang, Phys. Rev. A 52, 4116 (1995).

${ }^{10}$ T. Tsang, M. A. Krumbügel, K. W. DeLong, D. N. Fittinghoff, and R. Trebino, Opt. Lett. 21, 1381 (1996).

${ }^{11}$ R. W. Boyd, Nonlinear Optics (Academic, Boston, 1992).

${ }^{12}$ M. Muller, J. Squier, K. R. Wilson, and G. J. Grakenhoff, J. Microsc. 191, 266 (1998).

${ }^{13}$ M. Bruel, MRS Bull. 23, 35 (1998).

${ }^{14}$ Q.-Y. Tong and R. W. Bower, MRS Bull. 23, 40 (1998).

${ }^{15}$ P. N. Saeta and A. C. Gallagher, J. Appl. Phys. 77, 4639 (1995).

${ }^{16}$ P. N. Saeta and A. C. Gallagher, Phys. Rev. B 55, 4563 (1997).

${ }^{17}$ Handbook of Optical Constants of Solids, edited by E. Palik (Academic, Orlando, 1985).

${ }^{18}$ T. Yamaguchi, M. Nasu, Z. T. Jiang, M. Tabe, and Y. Kanda, Thin Solid Films 313, 264 (1998).

${ }^{19}$ D. J. Moss, H. M. van Driel, and J. E. Sipe, Opt. Lett. 14, 57 (1989).

${ }^{20}$ G. Lüpke, D. J. Bottomley, and H. M. van Driel, J. Opt. Soc. Am. B 11, 33 (1994).

${ }^{21}$ Details of the calculation will be published elsewhere.

${ }^{22}$ N. Bloembergen and P. S. Pershan, Phys. Rev. 128, 606 (1962).

${ }^{23}$ T. F. Heinz, in Nonlinear Surface Electromagnetic Phenomena, edited by H. E. Ponath and G. Stegeman (North-Holland, Amsterdam, 1991), Vol. 29, pp. 353-416. 